\title{
BOUNDARY VALUE AND EXPANSION PROBLEMS OF ORDINARY LINEAR DIFFERENTIAL EQUATIONS*
}

BY

\author{
GEORGE D. BIRKHOFF
}

\section{Introduction.}

Let $p_{2}(x), p_{3}(x), \cdots, p_{n}(x)$ be functions of the real variable $x$ on the closed interval $(a, b)$, which are continuous with their derivatives of all orders. Write

$$
L(z) \equiv \frac{d^{n} z}{d x^{n}}+*+p_{2}(x) \frac{d^{n-2} z}{d x^{n-2}}+\cdots+p_{n}(x) z,
$$

$$
M(z) \equiv(-1)^{n} \frac{d^{n} z}{d x^{n}}+*+(-1)^{n-2} \frac{d^{n-2}}{d x^{n-2}}\left[p_{2}(x) z\right]+\cdots+p_{n}(x) z .
$$

With the linear differential equation of the $n$th order in $u$

$$
L(u)+\lambda u=0 \dagger
$$

and $n$ linear homogeneous conditions in $u(a), u^{\prime}(a), \ldots, u^{(n-1)}(a), u(b)$, $u^{\prime}(b), \cdots, u^{(n-1)}(b)$,

$$
W_{1}(u)=0, W_{2}(u)=0, \cdots, W_{n}(u)=0
$$

we associate the like adjoint differential equation and $n$ like adjoint conditions

$$
M(v)+\lambda v=0
$$

$$
V_{1}(v)=0, V_{2}(v)=0, \cdots, V_{n}(v)=0 .
$$

For certain characteristic values of the complex parameter $\lambda$ there will exist

* The second part of a paper presented to the Society (Chicago), March 30, 1907, under a different title. The first part of this paper has been printed on pages 219-231 of this volume. Received for publication May 12, 1908.

$T$ It is not an essential generalization to write instead of (2)

$$
p_{0}(x) \frac{d^{n} z}{d x^{n}}+p_{1}(x) \frac{d^{n-1} z}{d x^{n-1}}+\cdots+p_{n}(x) z+\lambda P(x) z=0
$$

if $p_{0}+0, P \neq 0$, and $p_{0} / P$ is real. We therefore restrict (2) as stated. Max Masos has treated a special case $n=2$ with the restriction $P \neq 0$ removed, by different methods : these Transaotions, vol. 8 (1907), p. 427. 
a solution * $u(x)$ of (2), (3) or $v(x)$ of (4), (5). These values are the same in both cases: let them be

with corresponding solutions

$$
\lambda_{1}, \lambda_{2}, \cdots
$$

$$
\begin{aligned}
& u_{1}(x), u_{2}(x), \cdots, \\
& v_{1}(x), v_{2}(x), \cdots .
\end{aligned}
$$

These functions $u_{i}(x), v_{i}(x)$ are such that

$$
\int_{a}^{b} u_{i}(x) v_{j}(x) d x=0
$$

This property leads us to the formal expansion of a given function $f(x)$ on $(a, b)$,

$$
f(x) \sim \sum_{i=1}^{\infty} \frac{\int_{a}^{b} f(x) v_{i}(x) d x}{\int_{a}^{b} u_{i}(x) v_{i}(x) d x} \cdot u_{i}(x)
$$

How are the characteristic values distributed in the $\lambda$-plane? What is the nature of the solutions $u_{i}(x), v_{i}(x)$ ? In what sense does the expansion represent $f(x)$ ? These are the ginestions considered in this paper.

We begin with the derivation of the formal properties of the boundary value problem $(\$ 1)$, and of the expression for the sum of $n$ terms of $(7)$ by means of a contour integral $(\S 2)$.

There follows the more intimate study which is based on certain facts concerning the asymptotic nature of the solutions of $(2)$ and of $(4)$ when $|\lambda|$ is large. These facts are derived as an application of my paper in a preceding number of these Transactions ( $\$ 3)$. The distribution of the numbers $\lambda_{i}$ and the nature of the expansion is then obtained ( $\$ 4$ and $\S 5$ ). Finally the contour integral is evaluated and the representation theorem proved $(\S 6)$. The expansion is found to behave like a Fourier series except in the vicinity of $x=a$ and $x=b$.

Lrouvilue was the first to introduce the notion of adjoint conditions in a special case $f$ and to consider the related expansion. The results of the present paper are known for the real self-adjoint case $n=2$. $\ddagger$ WeSTFALL has proved a representation theorem for the real self-adjoint case $n=2 m$, providing that $f(x)$ and its first $n$ derivatives are continuous. $\S$

* By a solution we mean always a solution not identically zero.

†Liouville's Journal, ser. 1, vol. 3 (1838), p. 561. Professor E. H. Moorr suggested to me the possibility of gen sralization.

† A. Knesex, Małhematibche Annalen, vol. 58 (1901), p. 81 ; Drxon, Proceedings of the London Mathematisal Society, ser. 2, vol. 3 (1905), p. 83.

\& W. D. A. WhstralL, Zur Theorie der Integralgleichungen, A. MYLLER, Gewöhnliche Differentialgleichungen höherer Ordnung in ihrer Beziehung zu den Integralgleichungen. Göttingen dissertations (1905 and 1906). Reference should also be made to HILBERT's antecedent papers on integral equations in the Göttinger Nachrichten for 1904. 


\section{§1. The formal nature of the boundary value problem.}

We must first make precise the adjoint conditions referred to.

Definition. Let $W_{1}(u), \ldots, W_{n}(u)$ be $n$ given linear forms in $u(a)$, $u^{\prime}(a), \cdots, u^{(n-1)}(a), u(b), u^{\prime}(b), \cdots, u^{(n-1)}(b)$ and $W_{n+1}(u), \cdots, W_{2 n}(u)$ be any $n$ further linear forms so chosen that $W_{1}, \ldots, W_{2 n}$ are linearly independent. Then in the identity

$$
\left.\int_{a}^{b} z L(y) d x \equiv P(y, z)\right|_{x=a} ^{x=b}+\int_{a}^{b} y M(z) d x
$$

where $\left.P(y, z)\right|_{\substack{x=b \\ x=a}}$ is a bilinear form in $y(a), y^{\prime}(a), \cdots, y^{(n-1)}(a), y(b)$, $y^{\prime}(b), \cdots, y^{(n-1)}(b)$ and $z(a), z^{\prime}(a), \cdots, z^{(n-1)}(a), z(b), z^{\prime}(b), \cdots, z^{(n-1)}(b)$, we can write

$$
\left.P(y, z)\right|_{x=a} ^{x=b} \equiv \sum_{i=1}^{2 n} W_{i}(y) V_{2 n-i}(z),
$$

in which $V_{i}(z)$ are linear in $z(a), z^{\prime}(a), \cdots, z^{(n-1)}(a), z(b), z^{\prime}(b), \cdots, z^{(n-1)}(b)$ and linearly independent. Then $V_{i}(v)=0(i=1,2, \ldots, n)$ are the adjoint conditions to the given conditions $W_{i}(u)=0(i=1,2, \cdots, n)$. Any set of conditions $\bar{W}_{i}(u)=0(i=1,2, \cdots, n)$ equivalent to $W_{i}(u)=0(i=1,2, \ldots, n)$ by linear combination, with any choice of $\bar{W}_{n+1}(u), \ldots, \bar{W}_{2 n}(u)$ leads to a set of adjoint conditions $\vec{V}_{i}(v)=0(i=1,2, \ldots, n)$ equivalent to $V_{i}(v)=0$ $(i=1,2, \cdots, n)$ by linear combination.

Conversely, given $V_{i}(v)=0(i=1,2, \cdots, n)$ we choose $\nabla_{n+1}, \cdots, V_{2 n}$ as above and find $W_{i}(u)=0(i=1,2, \ldots, n)$ to be the adjoint conditions. Hence if the problem (4), (5) is adjoint to (2), (3), so also is (2), (3) adjoint to (4), (5).

The properties which this section proves are stated in I-III.

I. If for $\lambda=\lambda^{*}$ a solution $u^{*}(x)$ of (2), (3) exists, a solution $v^{*}(x)$ of (4), (5) will also exist for $\lambda=\lambda^{*}$; if $u^{*}(x)$ is unique (except for a constant factor), $v^{*}(x)$ is also unique (except for a constant factor).

Let $u^{\bullet}$ be the given solution of (2), (3) for $\lambda=\lambda^{*}$. Then we have

and for some $j$

$$
W_{1}\left(u^{*}\right)=W_{2}\left(u^{*}\right)=\cdots=W_{n}\left(u^{*}\right)=0 \text {, }
$$

as otherwise we should infer

$$
W_{n+j}\left(u^{*}\right) \neq 0 \text {, }
$$

$$
u^{\bullet}(a)=u^{\prime \prime}(a)=\cdots=u^{*(n-1)}(a)=u^{*}(b)=u^{* \prime}(b)=\cdots=u^{*(n-1)}(b)=0 \text {. }
$$

In the $n$-fold linear spread of solutions $v(x)$ of $(4)$ at $\lambda=\lambda^{*}$ there will be at least one, say $v^{\circ}(x)$, which satisfies the $n-1$ linear homogeneous conditions

$$
V_{n-i}\left(v^{*}\right)=0
$$

* Ce. SChlmsinger : Handbuch der Theorie der linearen Differentialgleichungen, vol. 1, p. 64, formula (1). 
Substitute in (8) $y=u^{*}, z=v^{*}$ and we obtain

$$
-\lambda^{*} \int_{a}^{b} v^{*} u^{*} d x=\dot{W}_{n+j}\left(u^{*}\right) \nabla_{n-j}\left(v^{*}\right)-\lambda^{*} \int_{a}^{b} u^{*} v^{*} d x \text {. }
$$

Therefore $V_{n-j}\left(v^{*}\right)=0$ also, and $v^{*}$ will satisfy (4), (5).

If $u^{*}$ is unique, $v^{*}$ is also unique. To prove this assume if possible that there were two linearly independent solutions $v^{*}$ and $v^{\text {"m }}$ satisfying (4), (5) when $\lambda=\lambda^{*}$, while $u^{*}$ is unique. Then we could choose $i$ and $j$ so that

$$
\left|\begin{array}{ll}
V_{n+i}\left(v^{*}\right) & V_{n+j}\left(v^{*}\right) \\
V_{n+i}\left(v^{* *}\right) & V_{n+j}\left(v^{* *}\right)
\end{array}\right| \neq 0 ;
$$

otherwise we should have constants $c, d$, not both zero, such that

$$
V_{i n+l}\left(c v^{*}+d v^{m}\right)=0 \quad(l=1,2, \cdots, n),
$$

and $v=c v^{*}+d v^{*}$ would fulfill the $2 n$ conditions

$$
V_{1}(v)=V_{2}(v)=\cdots=V_{2 n}(v)=0,
$$

which is not possible since the $V_{1}, \ldots, V_{2 n}$ are linearly independent. Choose now $u^{\prime \prime}$ linearly independent of $u^{*}$ to fulfill the $n-2$ conditions

$$
W_{l}\left(u^{* *}\right)=0, \quad(l \neq n-i \text { or } n-j)
$$

where $l=1,2, \ldots, n$. Writing in (8) $y=u^{* *}, z=v^{*}$ and $y=u^{* *}, z=v^{\prime \prime}$, we obtain, on simplifying,

$$
\begin{aligned}
& W_{n-i}\left(u^{m *}\right) V_{n+i}\left(v^{*}\right)+W_{n-j}\left(u^{\prime \prime}\right) V_{n+j}\left(v^{*}\right)=0, \\
& W_{n-i}\left(u^{m *}\right) V_{n+i}\left(v^{\prime \prime}\right)+W_{n-j}\left(u^{m *}\right) V_{n+j}\left(v^{* *}\right)=0 .
\end{aligned}
$$

From this we deduce that also

$$
W_{n-i}\left(u^{m}\right)=W_{n-j}\left(u^{m *}\right)=0 .
$$

Therefore $u^{\prime \prime}$ would satisfy (2), (3). This is impossible. Hence if $u^{*}$ is unique, $v^{*}$ is also unique, which we were to show.

Definition. If $\lambda=\lambda^{*}$ is a characteristic value of $\lambda$ for which one and but one solution of (2), (3) and (4), (5) exists, $\lambda^{*}$ is said to be a simple characteristic value.

II. If $y_{1}, y_{2}, \cdots, y_{n}$ are $n$ linearly independent solutions of (2) at $\lambda=\lambda^{*}$, the condition that $\lambda^{*}$ is a characteristic value is that the determinant

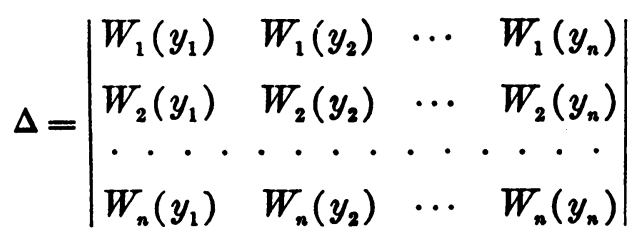


vanishes; the condition that $\lambda^{*}$ is a simple characteristic value is that not all the first minors vanish.

The general solution of $(2)$ is

$$
u=c_{1} y_{1}+c_{2} y_{2}+\cdots+c_{n} y_{n} \text {. }
$$

Under the condition $\Delta=0$ at least one determination of $c_{1}, c_{2}, \cdots, c_{n}$ is possible such that

$$
W_{1}(u)=0, W_{2}(u)=0, \cdots, W_{n}(u)=0 .
$$

This determination is unique when not all the first minors of $\Delta$ vanish.

III. If $u_{i}(x)$ and $v_{j}(x)$ belong to distinct characteristic numbers $\lambda_{i}$ and $\lambda_{j}$, then is

$$
\int_{a}^{b} u_{i} v_{j} d x=0
$$

For write $y=u_{i}, z=v_{j}$ in (8). Because of (9) we deduce

$$
-\lambda_{i} \int_{a}^{b} v_{j} u_{i} d x=-\lambda_{j} \int_{a}^{b} u_{i} v_{j} d x
$$

whence the desired relation follows.

In important particular cases the problem (4), (5) may be precisely (2), (3) with $v$ in place of $u$. We then say that the problem (2), (3) is self-adjoint. Or (4), (5) may be precisely (2), (3) with $v$ in place of $u$, except that $M(v)$ is the negative of $L(v)$. We then say that the problem (2), (3) is anti-self-adjoint.

\section{§2. Expansion as contour integral.}

It is easy to prove that when $\lambda$ is not a characteristic number there exists a unique $G(x, s ; \lambda) *$ such that the solution $\phi$ of

$$
L(\phi)+\lambda \phi=\omega, \quad W_{1}(\phi)=W_{2}(\phi)=\cdots=W_{n}(\phi)=0,
$$

is given by

$$
\phi=\int_{a}^{b} G(x, s ; \lambda) \omega(s) d s ;
$$

and likewise that there exists a unique $H(x, s ; \lambda)$ such that the solution $\phi$ of

$$
M(\phi)+\lambda \phi=\omega, \quad V_{1}(\phi)=V_{2}(\phi)=\cdots=V_{n}(\phi)=0,
$$

is given by

$$
\phi=\int_{a}^{b} H(x, s ; \lambda) \omega(s) d s .
$$

\footnotetext{
* Professor Bôchrr has defined the Green's function $G$ for an ordinary linear differential equation of order $n$ under a special form of conditions, and has stated the principal properties in the Bulletin of the American Mathematical Society, vol. 7 (1901), p. 297.
} 
The explicit formula for $G$ is

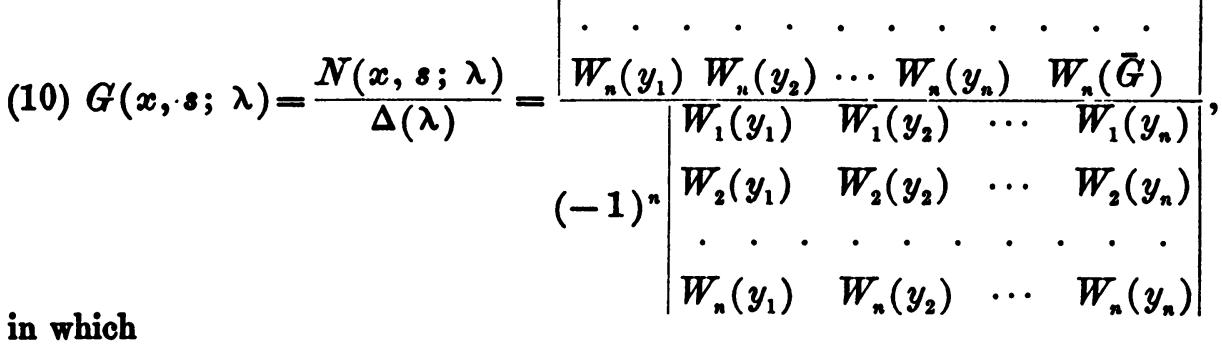

$$
\begin{array}{ccccc}
y_{1}(x) & y_{2}(x) & \cdots & y_{n}(x) & \bar{G}(x, s ; \lambda) \\
W_{1}\left(y_{1}\right) & W_{1}\left(y_{2}\right) & \cdots & W_{1}\left(y_{n}\right) & W_{1}(\bar{G}) \\
W_{2}\left(y_{1}\right) & W_{2}\left(y_{2}\right) & \cdots & W_{2}\left(y_{n}\right) & W_{2}(\bar{G})
\end{array}
$$

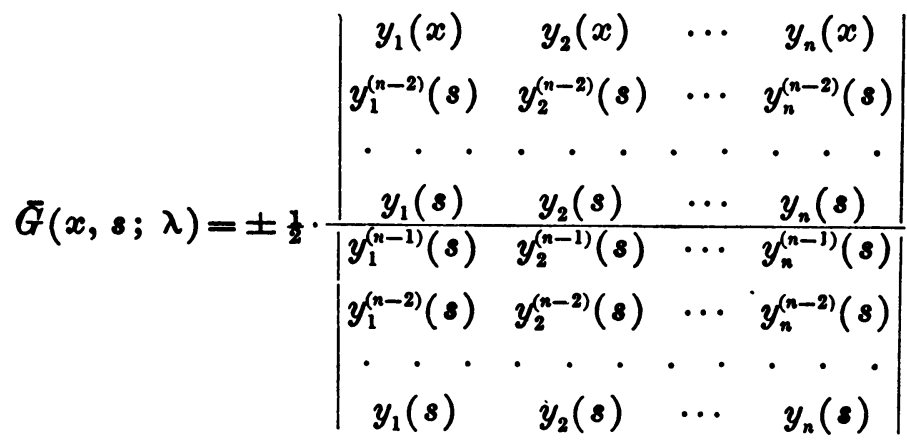$$
\text { (+ if } x>8,- \text { if } x<s \text { ). }
$$

Here $y_{1}, y_{2}, \cdots, y_{n}$ are any $n$ linearly independent solutions of (2). There is a like formula for $H(x, s ; \lambda)$. Furthermore

$$
G(x, s ; \lambda)=H(s, x ; \lambda) .^{*}
$$

The function $G$ is analytic in $\lambda$ (since $y_{1}, y_{2}, \ldots, y_{n}$ may be taken analytic in $\lambda$ ) except for a possible pole when $\Delta(\lambda)=0$, i. e. by II, $\S 1$, when $\lambda$ is a characteristic value.

If $\lambda=\lambda_{i}$ is a simple characteristic number for which $G$ has a pole of the first order, the residue is

where

$$
\frac{u_{i}(x) v_{i}(s)}{\int_{a}^{b} u_{i}(x) v_{i}(x) d x},
$$

Write

$$
\int_{a}^{b} u_{i}(x) v_{i}(x) d x \neq 0 \text {. }
$$

$$
G(x, s ; \lambda)=\frac{R(x, s)}{\lambda-\lambda_{i}}+\sigma(x, s ; \lambda),
$$

\footnotetext{
* For formulas like these and their prools see WrastraLL's dissertation, 8 8, 7.
} 
where $\sigma(x, s ; \lambda)$ is analytic at $\lambda=\lambda_{i}$. In view of $(10)$ we have

$$
R(x, s)=\frac{N\left(x, s ; \lambda_{i}\right)}{\Delta^{\prime}\left(\lambda_{i}\right)}
$$

where $N$ is continuous with its first $n$ derivatives since the coefficient of $\bar{G}$ is zero. Further $N$ satisfies (2), (3) for all $\lambda$ so that $R(x, s)$ must be a solution of (2), (3) in $x$. Because of the relation between $G$ and $H$, we infer also that $R(x, s)$ is a solution of $(4),(5)$ in $s$. By the definition of a simple characteristic value it follows that the residue is

$$
R(x, s)=c_{i} u_{i}(x) v_{i}(s) . *
$$

It remains to determine $c_{i}$.

By (12) we have

whence

$$
\lim _{\lambda=\lambda_{i}}\left[\left(\lambda-\lambda_{i}\right) G(x, s ; \lambda)-c_{i} u_{i}(x) v_{i}(s)\right]=0,
$$

$$
\lim _{\lambda=\lambda_{4}}\left[\left(\lambda-\lambda_{i}\right) \int_{a}^{b} G(x, s ; \lambda) u_{i}(s) d s-c_{i} u_{i}(x) \int_{a}^{b} v_{i}(s) u_{i}(s) d s\right]=0 .
$$

But since

$$
L\left(u_{i}\right)+\lambda u_{i}=\left(\lambda-\lambda_{i}\right) u_{i}, \quad W_{1}\left(u_{i}\right)=W_{2}\left(u_{i}\right)=\cdots=W_{n}\left(u_{i}\right)=0,
$$

we have by the fundamental property of $G$

$$
\left(\lambda-\lambda_{i}\right) \int_{a}^{b} G(x, s ; \lambda) u_{i}(s) d s=u_{i}(x) .
$$

Substituting this value above we find

Therefore

$$
\lim _{\lambda=\lambda_{i}}\left[u_{i}(x)-c_{i} u_{i}(x) \int_{a}^{b} v_{i}(s) u_{i}(s) d s\right]=0 .
$$

$$
c_{i} \int_{a}^{b} v_{i}(s) u_{i}(s) d s=1,
$$

out of which $c_{i}$ is determined; this proves that the rasidue has the stated form.

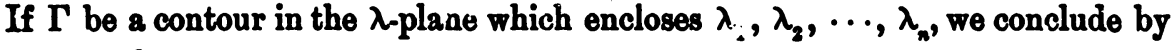
the above that

$$
\frac{1}{2 \pi \sqrt{-1}} \int_{\Gamma} \int_{a}^{b} G(x, s ; \lambda) f(s) d s d \lambda=\sum_{i=1}^{n} \frac{\int_{a}^{b} f(x) v_{i}(x) d x}{\int_{a}^{b} u_{i}(x) v_{i}(x) d x} \cdot u_{i}(x) .
$$

* In thir proof certain points of logio are obviously slurred orer. 
This is the sum of $n$ terms of the formal expansion, providing that $\lambda_{1}, \lambda_{2}, \ldots, \lambda_{n}$ are simple characteristic numbers at which $G$ has a pole of the first order. At values $\lambda_{i}$ for which these conditions are not satisfied, the corresponding term of the formal expansion (7) is to be replaced by

$$
\int_{a}^{b} R_{i}(x, s) f(s) d s
$$

where $R_{i}(x, s)$ is the residue. Thus in all cases we have an expansion each term of which is uniquely determined. We omit the development of $R_{i}(x, s)$ in these more complicated cases.

§3. The solutions of $L(u)+\lambda u=0$ and $M(v)+\lambda v=0$ when $|\lambda|$ is large.

As a preliminary to the deeper study of the problems which have been presented, I apply the results of my paper printed on pages 219-231 of the present volume to the differential equations $L(u)+\lambda u=0$ and $M(v)+\lambda v=0$.

If we write $\lambda=\rho^{n}$, the first of these differential equations can be written in the form there treated [see (1), loc. cit.],

$$
\begin{aligned}
& \frac{d^{n} u}{d x^{n}}+\rho a_{n-1}(x, \rho) \frac{d^{n-1} u}{d x^{n-1}}+\cdots+\rho^{n} a_{0}(x, \rho) u=0, \\
& a_{n-1}(x, \rho)=0, a_{n-2}(x, \rho)=\frac{p_{2}(x)}{\rho^{2}}, \cdots, a_{0}(x, \rho)=1+\frac{p_{n}(x)}{\rho^{n}}
\end{aligned}
$$

The coefficients obviously satisfy the restriction imposed, and the equation for $w_{i}(x)$ [see (4), loc. cit.], is

$$
w_{i}^{n}+1=0
$$

Thus $w_{1}, w_{2}, \ldots, w_{n}$ are constants. We also find (proof of Lemma 1, loc. cit.)

$$
u_{i 0}(x)=1 \quad(i=1,2, \cdots, n) .
$$

In order to state in explicit form the theorem for this case, it remains to consider the regions $S$ (definition, p. 220). Inasmuch as $w_{1}, w_{2}, \cdots, w_{n}$ are constants we can for each $\rho$ choose the indices 1 to $n$ so that

$$
R\left(\rho w_{1}\right) \leqq R\left(\rho w w_{2}\right) \leqq \cdots \leqq R\left(\rho w_{n}\right)
$$

for every $x$ on $(a, b)$. Hence every $\rho$ is on some region $S, \theta \leqq \arg \rho \leqq \psi$. At the bounding rays the ordering changes so that for some $i$ and $j$ 


$$
R\left(\rho w_{i}\right)=R\left(\rho r w_{j}\right)
$$

An easy computation shows that the regions $S$ are the regions

$$
\frac{l \pi}{n} \leqq \arg \rho \leqq \frac{(l+1) \pi}{n} \quad(l=0,1, \ldots, 2 n-1) .
$$

The differential equation $M(v)+\lambda v=0$ also is of the same type when we put $\lambda=\rho^{n}$. The roots $w_{i}(x)$ are

$$
-w_{1},-w_{2}, \cdots,-w_{n}
$$

and in the formal solutions we have

$$
u_{i 0}(x)=1 \quad(i=1,2, \cdots, n) .
$$

The regions $S$ are the same as for $L(u)+\lambda u=0$.

Let $E$ be a generic notation for functions of $\rho$ (and other variables), bounded when $|\rho|$ is large. The application of the theorem referred to gives:

On any region of the p-plane

$$
S: \quad \frac{l \pi}{n} \leqq \arg \rho \leqq \frac{(l+1) \pi}{n}
$$

there exist $n$ independent solutions,

$$
\begin{gathered}
y_{1}, y_{2}, \cdots, y_{n} \text { of } L(u)+\rho^{n} u=0, \\
z_{1}, z_{2}, \cdots, z_{n} \text { of } M(v)+\rho^{n} v=0,
\end{gathered}
$$

analytic in $\rho$ and such that on this region

where

$$
\begin{aligned}
& \left\{\begin{aligned}
& y_{i}=u_{i}(x, \rho)+e^{\rho w_{i}(x-a)} \frac{E_{0}}{\rho^{m}}, \\
& \frac{d y_{i}}{d x}=\frac{d}{d x} u_{i}(x, \rho)+e^{\rho w_{i}(x-a)} \frac{E_{1}}{\rho^{m-1}} \\
& \cdot \cdot \cdot \cdot \cdot \cdot \cdot \cdot \cdot \cdot \cdot \\
& \frac{d^{n-1} y_{i}}{d x^{n-1}}=\frac{d^{n-1}}{d x^{n-1}} u_{i}(x, \rho)+e^{\rho w_{i}(x-a)} \frac{E_{n-1}}{\rho^{m-n+1}},
\end{aligned}\right.
\end{aligned}
$$

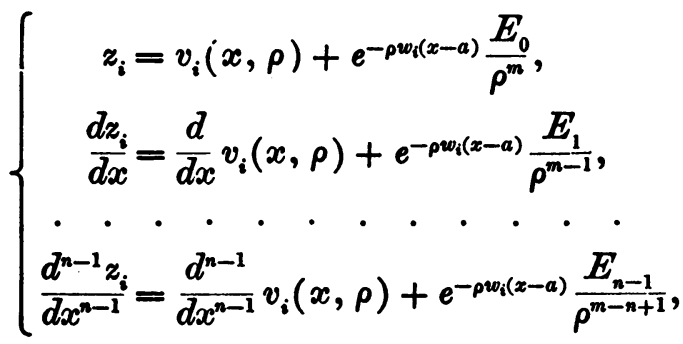




$$
\begin{aligned}
& u_{i}(x, \rho)=e^{\rho w_{i}(x-a)}\left[1+\frac{u_{i 1}(x)}{\rho}+\cdots+\frac{u_{i m}(x)}{\rho^{m}}\right], \\
& v_{i}(x, \rho)=e^{-\rho w_{i}(x-a)}\left[1+\frac{v_{i 1}(x)}{\rho}+\cdots+\frac{v_{i m}(x)}{\rho^{m}}\right],
\end{aligned}
$$

$w_{1}, w_{2}, \cdots, w_{n}$ being the $n$ roots of $w^{n}+1=0, m$ any positive integer.

\section{§4. Distribution of the characteristic values.}

The condition that $\lambda^{*}$ is a characteristic number is that the determinant $\Delta$ vanishes (II, $\S 1)$. The $i$-th element in the $j$-th column of $\Delta$ is $W_{i}\left(y_{j}\right)$ where $y_{1}, y_{2}, \cdots, y_{n}$ are any $n$ linearly independent solutions of $L(u)+\lambda^{*} u=0$. In order to treat the equation $\Delta=0$ we take $y_{i}$ to be the $y_{i}$ of $(20) \S 3$ where $\lambda=\rho^{n}$. In addition we assume the conditions $W_{i}$ to be normalized as follows. Reduce the number of conditions $W_{i}(u)=0$ of order $n-1$ [i. e., containing either $u^{(n-1)}(a)$ or $\left.u^{(n-1)}(b)\right]$ to a minimum, at most 2 , by linear combination. Then, in those that remain reduce the number of order $n-2$ to a minimum, at most 2 , by linear combination. Continue in this way as long as conditions remain. The normalized conditions will have the form

$$
\begin{aligned}
& W_{i}(u) \equiv W_{i a}(u)+W_{i b}(u)=0, \\
& W_{i a}(u) \equiv \alpha_{i} u^{\left(k_{i}\right)}(a)+\sum_{j=0}^{k_{i}-1} \alpha_{i j} u^{(j)}(a), \\
& W_{i b}(u) \equiv \beta_{i} u^{\left(k_{i}\right)}(b)+\sum_{j=0}^{k_{j}-1} \beta_{i j} u^{(j)}(b), \quad\left(k_{1} \geqq k_{3} \geqq \cdots \geqq k_{n}\right),
\end{aligned}
$$

in which no three successive $k$ 's are equal.

Definition. Let the $w_{i}$ be taken in every order such that for some $\rho \neq 0$

$$
R\left(\rho w_{1}\right)<R\left(\rho w_{2}\right)<\cdots<R\left(\rho w_{n}\right) .
$$

If $n=2 \mu-1$ and always neither $\theta_{0}=0$ nor $\theta_{1}=0$, where $\theta_{0}$ and $\theta_{1}$ are defined by the identity

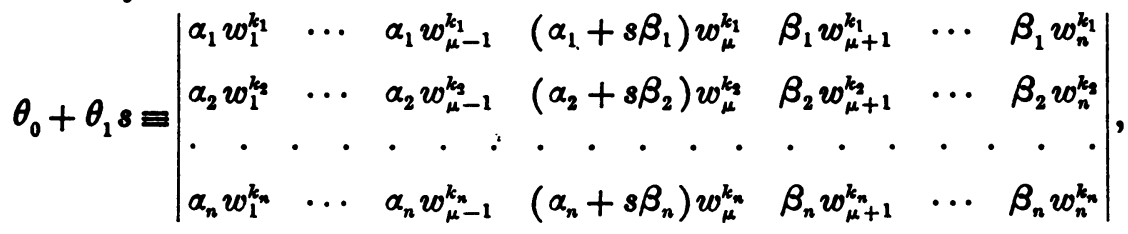

or if $n=2 \mu$ and always neither $\theta_{1}=0$ nor $\theta_{2}=0$, where $\theta_{0}, \theta_{1}$, and $\theta_{2}$ are defined by the identity 
(26)

$$
\begin{aligned}
& \theta_{0}+\theta_{1} s+\frac{\theta_{2}}{s} \equiv
\end{aligned}
$$

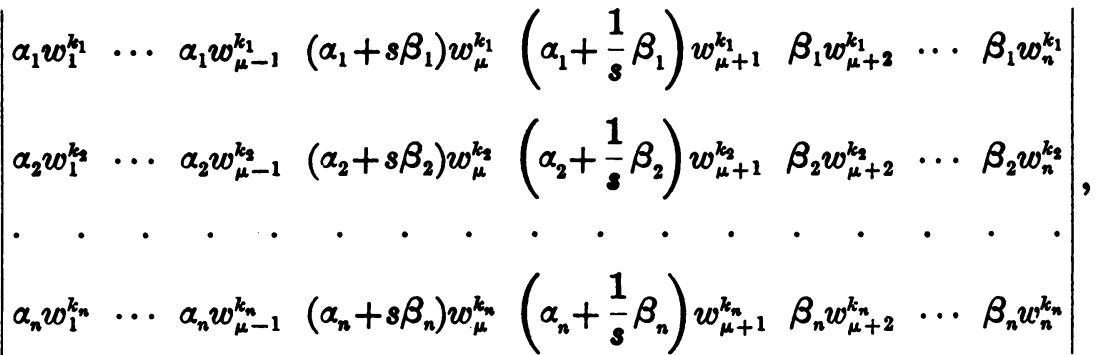

the conditions $W_{i}(u)=0(i=1,2, \cdots, n)$ are said to be regular.*

We now proceed to prove the

TheOREM. If the conditions $W_{i}(u)=0(i=1,2, \ldots, n)$ are regular, the characteristic values of $\lambda$ for (2), (3) and (4), (5) are in general simple $\dagger$ and form a pair of infinite sequences $\lambda_{I l}, \lambda_{\mathrm{II} l}(l=1,2, \ldots)$ such that

$$
\begin{aligned}
& \lambda_{\mathrm{Il} l}=-\left(\frac{2 l \pi \sqrt{-1}}{b-a}\right)^{n}\left(1+\sum_{j=1}^{m-1} \frac{g_{\mathrm{Ij}}}{l^{j}}+\frac{E_{\mathrm{il}}}{l^{m}}\right), \\
& \lambda_{\mathrm{IIl} l}=-\left(\frac{-2 l \pi \mathrm{l} \cdot \overline{-1}}{b-a}\right)^{n}\left(1+\sum_{j=1}^{m-1} \frac{g_{\mathrm{IIj}}}{l^{j}}+\frac{E_{\mathrm{IIl} l}}{l^{m}}\right),
\end{aligned}
$$

where $g_{\mathrm{IJ}}, g_{\mathrm{II} j}$ are constants and $\left|E_{\mathrm{Il}}\right|<M,\left|E_{\mathrm{II}}\right|<M$.

We consider the equation $\Delta=0$ in the $\rho$-plane, fixing attention on some region (19) for $l=l_{0}$. The transformation $\lambda=\rho^{n}$ makes two adjacent regions $S$ correspond to the entire $\lambda$-plane. It is convenient to consider separately the cases $n=2 \mu-1$ and $n=2 \mu$. The starting point is the set of relations [deduced from (21)]

$$
\begin{aligned}
& W_{i a}\left(y_{j}\right)=\left(\rho w_{j}\right)^{k_{i}}\left[\alpha_{i}+\sum_{l=1}^{m-1} \frac{\alpha_{i j l}}{\rho^{l}}+\frac{E}{\rho^{m}}\right], \\
& W_{i b}\left(y_{j}\right)=e^{\rho v v_{j}(b-a)}\left(\rho w_{j}\right)^{k_{i}}\left[\beta_{i}+\sum_{l=1}^{m-1} \frac{\beta_{i j l}}{\rho^{l}}+\frac{E}{\rho^{m}}\right] .
\end{aligned}
$$

* This apparently gives one condition for each region $S$, but these reduce to two for $n=2 \mu-1$ and to one when $n=2 \mu$. It is worthy of note that the STURM-LrouvinLE boundary conditions $(n=2)$ of the form

$$
h u(a)+k u^{\prime}(a)=0, \quad l u(b)+m u^{\prime}(b)=0,
$$

and also the periodio boundary conditions $(n=2)$

$$
u(a)=u(b), \quad u^{\prime}(a)=u^{\prime}(b),
$$

are regular. An example of non-regular conditions $(n=2)$ is

† See \$ 1.

$$
u(a)=0, \quad u^{\prime}(a)=k u(b) .
$$


CASE 1. $n=2 \mu-1$.

By definition of the regions $S$ we can choose indices 1 to $n$ so that

$$
R\left(\rho w_{1}\right) \leqq R\left(\rho w_{2}\right) \leqq \cdots \leqq R\left(\rho w w_{n}\right)
$$

for the region (19) under consideration. Making this choice we find readily that on $S$

while

$$
\begin{array}{lr}
R\left(\rho w_{i}\right)<0 & (i=1,2, \cdots, \mu-1), \\
R\left(\rho w w_{i}\right)>0 & (i=\mu+1, \mu+2, \cdots, n),
\end{array}
$$

$$
R\left(\rho w_{\mu}\right)=0, \quad \arg \rho=\frac{\left(l_{0}+\frac{1}{2}\right)}{n} \pi .
$$

From (28) and (29) we see that in the formula

$$
W_{i}\left(y_{j}\right)=W_{i a}\left(y_{j}\right)+W_{i b}\left(y_{j}\right) \quad(j=1,2, \cdots, \mu-1),
$$

the term $W_{i b}\left(y_{j}\right)$ can be absorbed into the term $W_{i a}\left(y_{j}\right)$ since $e^{\rho w_{j}(b-a)}$ is small of an infinite order in $1 / \rho$ on $S$; that is, we can write

$$
W_{i}\left(y_{j}\right)=\left(\rho w_{j}\right)^{k_{i}}\left[\alpha_{i}+\sum_{i=1}^{m-1} \frac{\alpha_{i j l}}{\rho^{l}}+\frac{E}{\rho^{m}}\right] \quad(j=1,2, \cdots, \mu-1) .
$$

By like reasoning we find

$$
W_{i}\left(y_{j}\right)=e^{\rho v_{j}(b-a)}\left(\rho w_{j}\right)^{k_{i}}\left[\beta_{i}+\sum_{l=1}^{m-1} \frac{\beta_{i j l}}{\rho^{l}}+\frac{E}{\rho^{m}}\right] \quad(j=\mu+1, \mu+2, \cdots, n) .
$$

Also from (28)

$$
\begin{aligned}
W_{i}\left(y_{\mu}\right)=\left(\rho w_{\mu}\right)^{k_{i}}\left[\alpha_{i}\right. & \left.+\sum_{l=1}^{m-1} \frac{\alpha_{i \mu l}}{\rho^{l}}+\frac{E}{\rho^{m}}\right] \\
& +e^{\rho w_{\mu}(b-a)}\left(\rho w_{\mu}\right)^{k_{i}}\left[\beta_{i}+\sum_{l=1}^{m-1} \frac{\beta_{i \mu l}}{\rho^{l}}+\frac{E}{\rho^{m}}\right] .
\end{aligned}
$$

If we substitute in $\Delta$ these values of $W_{i}\left(y_{j}\right)$ as given in (31), (32), (33), and remove the factors $\rho^{k_{i}}$ from the $i$-th row $(i=1,2, \ldots, n)$, and the factors $e^{e^{p p_{j}(b-a)}}$ from the $j$-th column $(j=\mu+1, \mu+2, \cdots, n)$, i. e., altogether the non-zero factor

the condition takes the form

$$
\prod_{i=1}^{n} \rho^{k_{i}} \cdot \prod_{j=\mu+1}^{n} e^{\rho w_{j}(b-a)}
$$

$$
\begin{gathered}
\bar{\Delta}=\left(\theta_{0}+\sum_{j=1}^{m-1} \frac{c_{j}}{\rho^{j}}+\frac{E_{0}}{\rho^{m}}\right)+e^{\rho w_{\mu}(b-a)}\left(\theta_{1}+\sum_{j=1}^{m-1} \frac{d_{j}}{\rho^{j}}+\frac{E_{1}}{\rho^{m}}\right)=0, \\
\Delta=\prod_{i=1}^{n} \rho^{k_{i}} \cdot \prod_{j=\mu+1}^{n} e^{\rho w_{j}(b-a)} \cdot \bar{\Delta} .
\end{gathered}
$$


The quantities $\theta_{0}$ and $\theta_{1}$ are the $\theta_{0}$ and $\theta_{1}$ of (25). In fact (25) was formed to give these terms.

Since $\Delta$ was analytic in $\rho, \bar{\Delta}$ will be also analytic in $\rho$.

The expressions of the first minors of the $\mu$-th column of $\bar{\Delta}$,

$$
a+\frac{b}{\rho}+\cdots+\frac{E}{\rho^{m}},
$$

zannot have all their first terms $a=0$ since then $\theta_{0}=\theta_{1}=0$, contradicting the hypothesis. Hence for large values of $|\rho|$ not all these first minors vanish. If we return to $\Delta$, we see that accordingly not all its first minors vanish. The characteristic numbers when $|\lambda|$ is large must therefore be simple. This was part of the theorem.

From (34) we obtain the equivalent condition

$$
e^{\rho w_{\mu}(b-a)}=\delta(\rho), \quad \delta(\rho)=-\frac{\theta_{0}}{\theta_{1}}+\sum_{j=1}^{m-1} \frac{\delta_{j}}{\rho^{j}}+\frac{E}{\rho^{m}}
$$

by solving for $e^{\rho \omega_{\mu}(b-a)}$. From this we infer

$$
\begin{aligned}
\rho w_{\mu}(b-a) & =\log \delta(\rho), \\
\log \delta(\rho) & =\log \left(-\frac{\theta_{0}}{\theta_{1}}\right)+\sum_{j=1}^{m-1} \frac{e_{j}}{\rho^{m}}+\frac{E}{\rho^{m}} \pm 2 l \pi \sqrt{-1},
\end{aligned}
$$

the final form of the condition, in which of course $E$ is analytic in $\rho$ on $S$.

In view of this condition, it is obvious that the values of $\rho$ on $S$ which satisfy $\Delta=0$ have when $|\rho|$ is large the form

$$
\rho_{l}= \pm \frac{2 l \pi \sqrt{-1}}{w_{\mu}(b-a)}+\frac{\log \left(-\frac{\theta_{0}}{\theta_{1}}\right)}{\omega_{\mu}(b-a)}+\sum_{j=1}^{m-1} \frac{h_{j}}{l^{j}}+\frac{E}{l^{m}},
$$

where $l$ is a large positive integer. Since by (30)

$$
\arg \omega_{\mu}=-\frac{\left(l_{0}+\frac{1}{2}\right)}{n} \pi \pm \frac{\pi}{2},
$$

we see from (36) that the \pm sign is to be so chosen that the $\rho_{l}$ approach asymptotically (in an angular sense) the bisecting ray of $S$. Let now $\rho$ describe a circle of fixed small radius $r$ about

$$
\pm \frac{2 l \pi \sqrt{-1}}{w_{\mu}(b-a)}+\frac{\log \left(-\frac{\theta_{0}}{\theta_{1}}\right)}{w_{\mu}(b-a)} .
$$

This will be wholly within $S$ when $|\rho|$ is large; and

$$
\arg \left[\rho \omega_{\mu}(b-a)-\log \delta(\rho)\right]
$$


increases by $2 \pi$ if the inequality

$$
|r(b-a)|>\left|\sum_{j=1}^{m-1} \frac{e_{j}}{\rho^{j}}+\frac{E}{\rho^{m}}\right|,
$$

obtains as is true for $|\rho|$ large. This proves the unique existence of $\rho_{l}$ when $|\rho|$ is large, and the distribution of values $\rho$ is determined.

It remains to return to the $\lambda$-plane; $S$ becomes $S^{\prime}$

$$
S^{\prime}: \quad l_{0} \pi \leqq \arg \lambda \leqq\left(l_{0}+1\right) \pi .
$$

The values of $\lambda$ approach the positive or negative axis of imaginaries according as $l_{0}$ is even or odd, and by (36) have the form stated in the theorem where for some $\theta_{0}, \theta_{1}$

$$
g_{\mathrm{I} 1} \text { or } g_{\mathrm{II} 1}= \pm \frac{n \log \left(-\frac{\theta_{0}}{\theta_{1}}\right)}{2 \pi \sqrt{-1}} \text {. }
$$

This completes the proof when $n=2 \mu-1$.

CASE II. $n=2 \mu$.

The proof is analogous to that for Case l. If $\rho w_{1}, \rho w_{2}, \cdots, \rho w_{n}$ have increasing real parts on $S$ we find

while

$$
\begin{array}{lr}
R\left(\rho w_{i}\right)<0 & (i=1,2, \cdots, \mu-1), \\
R\left(\rho w_{i}\right)>0 & (i=\mu+2, \mu+3, \cdots, n),
\end{array}
$$

$$
R\left(\rho w_{\mu}\right)=R\left(\rho w w_{\mu+1}\right)=0, \quad \arg \rho=\operatorname{either} \frac{l_{0} \pi}{n} \text { or } \frac{\left(l_{0}+1\right) \pi}{n},
$$

and $w_{\mu+1}=-w_{\mu}$. The region $S$ is of course defined by (19) as before. We now find $W_{i}\left(y_{j}\right)$ to be of the form (31) for $j=1,2, \ldots, \mu-1$ and of the form (32) for $j=\mu+2, \mu+3, \cdots, n$; also for $j=\mu, \mu+1$ we find

$$
W_{i}\left(y_{: L}\right)=\left(\rho w w_{\mu}\right)^{k_{i}}\left[\alpha_{i}+\sum_{l=1}^{n-1} \frac{\alpha_{i \mu l}}{\rho^{l}}+\frac{E}{\rho^{m}}\right]+e^{\rho w_{\mu}(b-a)}\left(\rho w_{\mu}\right)^{k_{i}}\left[\beta_{i}+\sum_{l=1}^{m-1} \frac{\beta_{i \mu l}}{\rho^{l}}+\frac{E}{\rho^{m}}\right],
$$

$W_{i}\left(y_{\mu+1}\right)=\left(-\rho w w_{\mu}\right)^{k_{i}}\left[\alpha_{i}+\sum_{l=1}^{m-1} \frac{\alpha_{i, \mu+1, l}}{\rho^{i}}+\frac{E}{\rho^{m}}\right]$

$$
+e^{-\rho w w_{\mu}(b-a)}\left(-\rho v w_{\mu}\right)^{k_{i}}\left[\beta_{i}+\sum_{l=1}^{m-1} \frac{\beta_{i, \mu}+1, l}{\rho^{l}}+\frac{E}{\rho^{m}}\right] \text {, }
$$

writing $w_{\mu+1}=-w_{\mu}$.

These expressions $W_{i}\left(y_{j}\right)$ are substituted in $\Delta$ and a factor

$$
\prod_{i=1}^{n} \rho^{k_{i}} \cdot \prod_{j=\mu+2}^{n} e^{\rho w_{j}(b-a)}
$$

is removed. The equation $\Delta=0$ becomes then of the form 


$$
\begin{gathered}
\bar{\Delta}=\left(\theta_{0}+\sum_{j=1}^{m-1} \frac{c_{j}}{\rho^{j}}+\frac{E_{0}}{\rho^{m}}\right)+e^{\rho w_{\mu}(b-a)}\left(\theta_{1}+\sum_{j=1}^{m-1} \frac{d_{j}}{\rho^{j}}+\frac{E_{1}}{\rho^{m}}\right) \\
\quad+e^{-\rho w_{\mu}(b-a)}\left(\theta_{2}+\sum_{j=1}^{m-1} \frac{e_{j}}{\rho^{j}}+\frac{E_{2}}{\rho^{m}}\right)=0, \\
\Delta=\prod_{i=1}^{n} \rho^{k_{i}} \cdot \prod_{j=\mu+2}^{n} e^{\rho w_{j}(b-a)} \cdot \bar{\Delta},
\end{gathered}
$$

$\theta_{0}, \theta_{1}, \theta_{2}$ being defined as in (26). The equation (38) is quadratic in $e^{\rho w_{\mu}(b-a)}$.

This equation (38) will yield the forms stated in the theorem for the characteristic values $\lambda_{1 l}, \lambda_{\mathrm{IIl}}$ on $S$. They must lie near to one of the bounding rays, say $\arg \rho=\left(l_{0}+1\right) \pi / n$. If we consider the necessary form of the expansion on the other region $S$ adjoining this ray, i. e., on the region

$$
\frac{\left(l_{0}+1\right) \pi}{n} \leqq \arg \rho \leqq \frac{\left(l_{0}+2\right) \pi}{n}
$$

we obtain the same equation (38) except for different $E$-terms perhaps; hence the same necessary form for $\lambda_{I l}, \lambda_{I l}$ as before. Thus we have the necessary form for the entire $\lambda$-plane.

The final fact of unique existence is demonstrated as before unless

$$
\text { either } \arg g_{\mathrm{I} 1}=\left\{\begin{array}{l}
0 \\
\pi
\end{array} \text { or } \quad \arg g_{\mathrm{II} 1}=\left\{\begin{array}{c}
0 \\
\pi
\end{array}\right.\right.
$$

when the values $\rho_{\mathrm{Il}}, \rho_{\mathrm{II} l}$ may come indefinitely near to the bounding ray $\arg \rho=\left(l_{0}+1\right) \pi / n$. By considering first the case

$$
p_{2}(x)=p_{3}(x)=\cdots=p_{n}(x)=0,
$$

when the solutions $y_{i}$ are known, and by using continuity considerations, the fact of unique existence can be established for this case also.

\section{§5. Nature of the expansion.}

In this section we give a notion of the character of $u_{i}, v_{i}$ and of the expan$\operatorname{sion}(7)$.

Let us first develop the character of $u_{I l}$ in the case $n=2 \mu-1$. Clearly

$$
u_{1 l}=c_{1} y_{1}+\cdots+c_{n} y_{n}
$$

where we have for the determination of $c_{1}, c_{2}, \cdots, c_{n}$ the equations

$$
c_{1} W_{i}\left(y_{1}\right)+c_{2} W_{i}\left(y_{2}\right)+\cdots+c_{n} W_{i}\left(y_{n}\right)=0 \quad(i=1,2, \cdots, n) .
$$

Substitute in the matrix $\left\|W_{i}\left(y_{j}\right)\right\|$ the expressions given in (31), (32), (33) and remove the factor $\rho^{k_{i}}$ from the $i$ th column $(i=1,2, \ldots, n)$; write for $\rho$ the values (36). This matrix then takes the form 


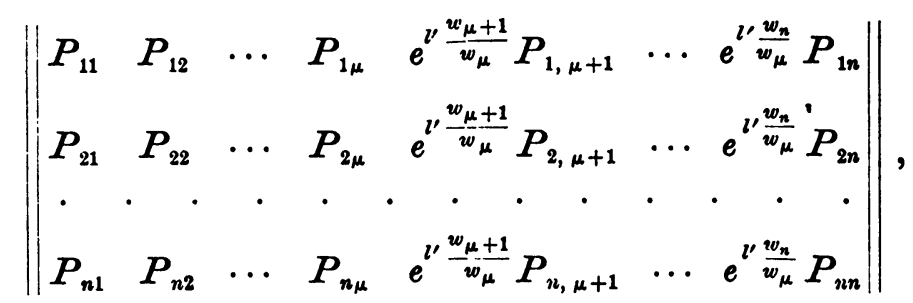

where the $P_{i j}$ are of the form

$$
a+\frac{b}{l}+\cdots+\frac{E}{l^{n}}
$$

and

$$
l^{\prime}=2 l \pi \sqrt{-1}+\frac{2 \pi v^{\prime} \overline{-1}}{n} g_{\mathrm{r} 1} .
$$

The $c_{i}$ are proportional to the minors of any row, say the first. The minors of the elements 1 to $\mu$ contain all the factors

$$
\prod_{i=\mu+1}^{n} e^{e^{\prime} \frac{w_{i}}{w_{\mu}}}
$$

besides a factor like (42), while the minors of the eler $\quad i>\mu$, contain all these except $e^{l / w_{v} w_{\mu}}$ besides a factor like (42). We conclude that

where $Q_{i}$ is of the form (42).

$$
\begin{aligned}
c_{i}=Q_{i} & (i=1,2, \cdots, \mu), \\
c_{i}=e^{-l^{\prime} \frac{w_{i}}{w_{\mu}}} Q_{i} & (i=\mu+1, \mu+2, \cdots, n),
\end{aligned}
$$

If we substitute into (21) the values (36), we obtain

where $Y_{i}$ is of the form

$$
\begin{aligned}
& y_{i}=e^{l^{\prime} \frac{w_{i}}{w_{\mu}}\left(\frac{x-a}{b-a}\right)} Y_{i} \quad(i=1,2, \cdots, n), \\
& a+\frac{b(x)}{l}+\cdots+\frac{E}{l^{m}} .
\end{aligned}
$$

Placing the expressions for $c_{i}$ and $y_{i}$ of (43) and (44) in (39), we derive the form of $u_{\mathrm{Il}}$; likewise the form of $u_{\mathrm{II} l}, v_{\mathrm{Il}}, v_{\mathrm{II} l}$ is obtained. We have finally

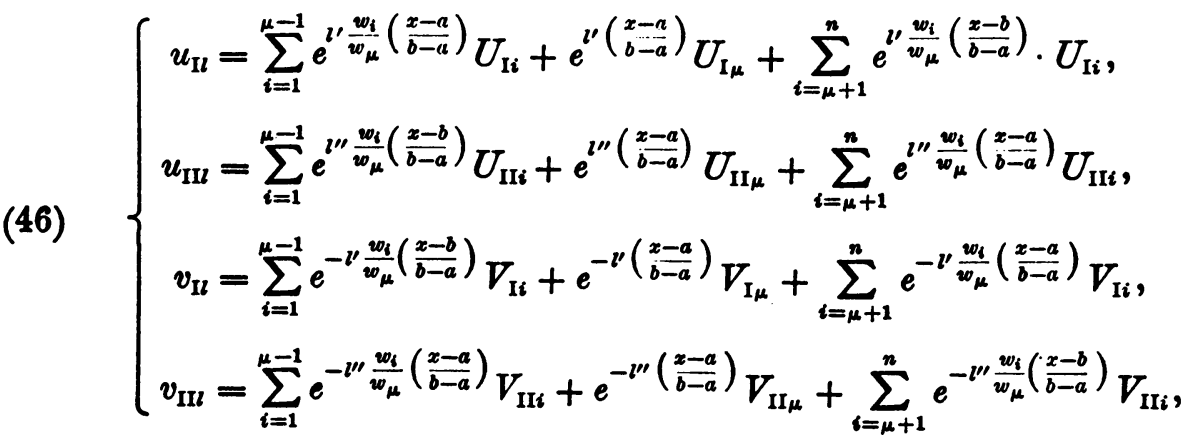




$$
l^{\prime}=2 l \pi \sqrt{-1}+\frac{2 \pi \sqrt{-1} g_{\mathrm{II}}}{n}, \quad l^{\prime \prime}=-2 l \pi \sqrt{\overline{-1}}-\frac{2 \pi \sqrt{-1} g_{\mathrm{II}}}{n}:
$$

Here $U, V$ are of the form (45) and $n=2 \mu-1$.

When $n=2 \mu$ we get in the same manner

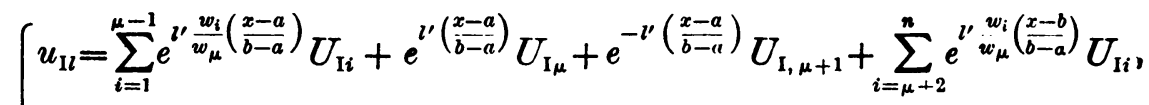

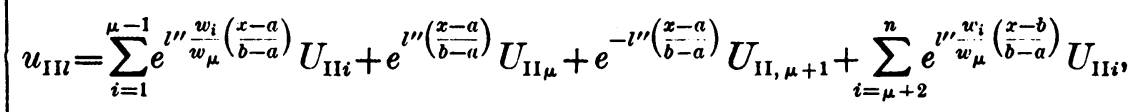

$$
\begin{aligned}
& \left\{v_{\mathrm{I} l}=\sum_{i=1}^{\mu-1} e^{-l^{\prime} \frac{w_{i}}{w_{\mu}}\left(\begin{array}{l}
x-b \\
b-a
\end{array}\right)} V_{\mathrm{I} i}+e^{-l^{\prime}\left(\begin{array}{c}
x-a \\
b-a
\end{array}\right)} V_{\mathrm{I} \mu}+e^{l^{\prime}\left(\begin{array}{c}
x-a \\
b-a
\end{array}\right)} V_{\mathrm{I}, \mu+1}+\sum_{i=\mu+2}^{n} e^{-l^{\prime} \frac{x_{i}}{w_{\mu}}\left(\begin{array}{c}
x-a \\
b-a
\end{array}\right)} V_{\mathrm{I} i},\right. \\
& v_{\mathrm{IIl}}=\sum_{i=1}^{\mu-1} e^{-l^{\prime \prime} \frac{w_{i}}{w_{\mu}}\left(\frac{x-b_{i}}{b-a}\right)} V_{\mathrm{II} i}+e^{-l^{\prime \prime \prime}\left(\begin{array}{l}
x-a \\
b-a
\end{array}\right)} V_{\mathrm{II} \mu}+e^{l \prime\left(\frac{x-a}{b-a}\right)} V_{\mathrm{II}, \mu+1} \\
& +\sum_{i=\mu+2}^{n} e^{-l{ }^{\prime \prime} \frac{w_{i}}{w_{\mu}}\left(\begin{array}{c}
x-a \\
b-a
\end{array}\right)} V_{\mathrm{II} i} \\
& l^{\prime}=2 l \pi \sqrt{-1}+\frac{2 \pi \sqrt{-1} g_{\mathrm{I} 1}}{n}, \quad l^{\prime \prime}=2 l \pi \sqrt{-1}+\frac{2 \pi V^{\prime}-1 g_{\mathrm{II}}}{n} .
\end{aligned}
$$

In both (46) and (47) it is to be noted that the $\Sigma$-terms are not important except at $x=a$ and $x=b$, since the real parts of the exponential terms are large and negative for $l$ large and $a<x<b$. We omit the fuller discussion, to be made by (46) and (47). It is worthy of remark that the simplest case

$$
n=1, \quad L(u)=u^{\prime}, \quad W_{1}(u)=u(a)-u(b)
$$

yields the Fourier series. The general expansion is clearly of a similar nature.

§6. Convergence of expansion to $f(x)$.

We have expressed the sum of $n$ terms of the expansion (7) in the form

$$
I=\frac{1}{2 \pi v-1} \int_{\Gamma} \int_{a}^{b} G(x, s ; \lambda) f(s) d s d \lambda,
$$

where $\Gamma$ is some contour which contains $\lambda_{1}, \lambda_{2}, \ldots, \lambda_{n}$ but no other characteristic values within it; $G$ is explicitly defined [see (10)] in terms of the solutions $y_{i}$ of $L(u)+\lambda u=0$. By means of the known asymptotic character of the $y_{i}$ we determine the character of $G$, and then evaluate the contour integral as $\Gamma$ enlarges without bound.

For convenience let us use the notation $[w]$ for an expression

$$
w+\frac{a}{\rho}+\cdots+\frac{E}{\rho^{m}},
$$

where $w, a, \cdots$ are independent of $\rho$ but need not be constants. 
Theorem. Let $f(x)$ be made up of a finite number of pieces in the interval $a \leqq x \leqq b$, each real, continuous, and with a continuous derivative. Unless $x=a$ or $x=b$, the expansion for $f(x)$ connected with the differ. ential equation $L(u)+\lambda u=0$ and the regular boundary conditions $W_{i}(u)=0(i=1,2, \cdots, n)$ converges to $[f(x+0)+f(x-0)] / 2$. At $x=a$ the series converges to

and at $x=b$ to

$$
a_{1} f(a+0)+a_{2} f(b-0)
$$

$$
b_{1} f(a+0)+b_{2} f(b-0) \text {, }
$$

where $a_{1}, a_{2}, b_{1}, b_{2}$ are constants independent of $f(x) .^{*}$

Proof. We restrict ourselves first to the case $n=2 \mu-1$. At the end we outline a similar proof for $n=2 \mu$.

Let the contour $\Gamma$ be taken as a circle $|\lambda|=k$ in the $\lambda$-plane. If the transformation $\lambda=\rho^{n}$ be made, we find

$$
I=\int_{\gamma_{1}} \int_{a}^{b} n \rho^{n-1} G(x, s ; \lambda) f(s) d s d \rho+\int_{\gamma_{\varepsilon}} \int_{a}^{b} n \rho^{n-1} G(x, s ; \lambda) f(s) d s d \rho .
$$

In this expression $\gamma_{1}$ is the segment of a circle $|\rho|=k^{\prime}$ lying on a region $S$,

$$
S_{1}: \quad \frac{l_{0} \pi}{n} \leqq \arg \rho \leqq \frac{\left(l_{0}+1\right) \pi}{n},
$$

and $\gamma_{2}$ is the segment of the same circle lying on the adjacent region $S$,

$$
S_{2}: \quad \frac{\left(l_{0}+1\right) \pi}{n} \leqq \arg \rho \leqq \frac{\left(l_{0}+2\right) \pi}{n} .
$$

We confine our attention to the partial integral

$$
I_{\gamma_{1}}^{a-x}=\int_{\gamma_{1}} \int_{a}^{x} n \rho^{n-1} G(x, s ; \lambda) f(s) d s d \rho,
$$

taking $a<x<b$. It is found to tend toward $f(x-0) / 4$. By considerations of symmetry the limiting values of the remaining partial integrals are determined. The first part of the theorem then follows.

Let $B$ be the bisection point of $\gamma_{1}$ and $A, C$ its end points. Take $A B$ as that segment of $\gamma_{1}$ for which

$$
R\left(\rho w_{\mu}\right)<0
$$

and $B C$ as that segment of $\gamma_{1}$ for which

$$
R\left(\rho w_{\mu}\right)>0 .
$$

* For definitions of regular conditions see $\S 4$. The restrictions imposed on $f(x)$ might be lightened but only at the cost of brevity. 
The indices 1 to $n$ of $w_{i}$ are chosen as in (29) and (30). We must break up $I_{\gamma_{1}}^{a-x}$ in two parts $I_{A B}^{a-x}$ and $I_{B C}^{a-x}$, both of which are attacked in the same way. To treat $G$ on $A B$, write

$$
\bar{G}(x, s ; \lambda)= \pm \frac{1}{2} \sum_{i=1}^{n} y_{i}(x) \bar{y}_{i}(s) \quad\left\{\begin{array}{l}
+ \text { if } x>s \\
- \text { if } x<s
\end{array}\right.
$$

where the $y_{i}$ of (11) have been taken as the $y_{i}$ of (21). Then $\bar{y}_{i}$ is the coeffcient of $\tau_{i}$ in

But by (21)

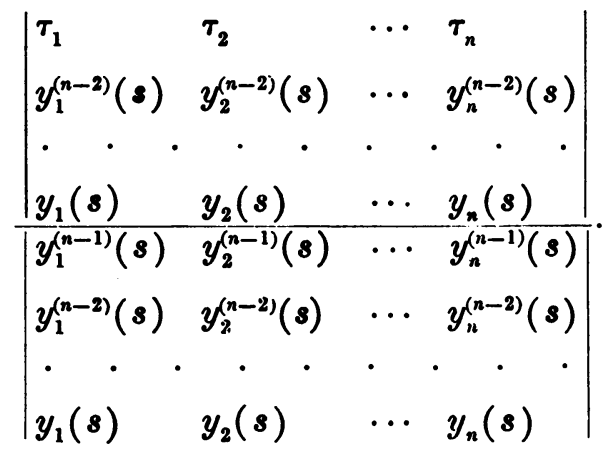

$$
y_{i}^{(j)}(s)=e^{\rho w(s-a)} \rho^{j}\left[w_{i}^{j}\right] .
$$

If these expressions be substituted above we find

$$
\bar{y}_{i}(s)=e^{-\rho w_{i}(s-a)} \frac{1}{n \rho^{n-1}}\left[-w_{i}\right]^{*}
$$

since $-w_{i} / n$ is the coefficient of $\tau_{i}$ in the expression

$$
\frac{\left|\begin{array}{llll}
\tau_{1} & \tau_{2} & \cdots & \tau_{n} \\
w_{1}^{n-2} & w_{2}^{n-2} & \cdots & w_{n}^{n-2} \\
\cdot & \cdot & \cdot & \cdot \\
1 & 1 & \cdots & 1
\end{array}\right|}{\left|\begin{array}{cccc}
w_{1}^{n-1} & w_{2}^{n-1} & \cdots & w_{n}^{n-1} \\
w_{1}^{n-2} & w_{2}^{n-2} & \cdots & w_{n}^{n-2} \\
\cdot & \cdot & \cdot & \cdot \\
1 & 1 & \cdots & 1
\end{array}\right|}
$$

* The denominator contains the faotors $e^{p v_{\mathrm{A}}(e-a)}$ whioh we divide into the correspondingfi-th columns of the numerator. It is to be remembered that, if $t+0$,

$$
\frac{1}{[t]}=\left[\frac{1}{t}\right] \text {. }
$$


In fact this coefficient $m_{i}$ satisfies the conditions

$$
\sum_{i=1}^{n} w_{i}^{j} m_{i}=0 \quad(j=0,1, \cdots, n-2) \quad \sum_{i=1}^{n} w_{i}^{n-1} m_{i}=1 .
$$

These show that $m_{i}$ has the stated value.

By (51)

$$
\bar{G}(x, s ; \lambda)=+\frac{1}{2} \sum_{i=1}^{n} y_{i}(x) \bar{y}_{i}(s)
$$

since $a<s<x$ for the partial integral $I_{A B}^{a-x}$. Also

$$
W_{i}(\bar{G})=-\frac{1}{2} \sum_{j=1}^{n} W_{i a}\left(y_{j}\right) \bar{y}_{j}(s)+\frac{1}{2} \sum_{j=1}^{n} W_{i b}\left(y_{j}\right) \bar{y}_{j}(s) .
$$

From these equations it is clear that if we multiply the columns 1 to $\mu$ of $N(x, s ; \lambda)$ in $(10)$ by $+\frac{1}{2} \bar{y}_{i}(s)(i=1,2, \ldots, \mu)$, the columns $\mu+1$ to $n$ by $-\frac{1}{2} \bar{y}_{i}(s)(i=\mu+1, \mu+2, \ldots, n)$ and add them to the last column, this last column has the elements

$$
+\sum_{i=1}^{\mu} y_{i}(x) \bar{y}_{i}(s), \quad \sum_{j=1}^{\mu} W_{i b}\left(y_{j}\right) \bar{y}_{j}(s)-\sum_{j=\mu+1}^{n} W_{i a}\left(y_{j}\right) \bar{y}_{j}(s) \quad(i=1,2, \cdots, n) .
$$

By (21), (28), and (53) these are of the forms

$$
\begin{gathered}
-\frac{1}{n \rho^{n-1}} \sum_{i=1}^{\mu} e^{\rho w_{i}(x-s)}\left[w_{i}\right], \\
\frac{1}{n \rho^{n-1}}\left(\sum_{j=1}^{\mu} e^{\rho w_{j}(b-s)} \rho^{k_{i}}\left[-\beta_{i} w_{j}^{k_{j}+1}\right]+\sum_{j=\mu+1}^{n} e^{\rho w_{j}(a-s)} \rho^{k_{i}}\left[\alpha_{i} w_{j}^{k_{i}+1}\right]\right)(i=1,2, \cdots, n) .
\end{gathered}
$$

In the new notation equations (21), (31), (32), (33), and (34) give also

$$
\begin{aligned}
& y_{i}=e^{\rho w_{i}(x-a)}[1] \\
& (i=1,2, \cdots, n), \\
& W_{i}\left(y_{j}\right)=\left\{\begin{array}{lr}
\rho^{k_{i}}\left[\alpha_{i} w_{j}^{k_{i}}\right] & (j=1,2, \cdots, \mu-1), \\
\rho^{k_{i}}\left(\left[\alpha_{i} w_{\mu}^{k_{i}}\right]+e^{\rho w_{\mu}(b-a)}\left[\beta_{i} w_{\mu}^{k_{i}}\right]\right) & (j=\mu), \\
\rho^{k_{i}} e^{\rho u_{j}(b-a)}\left[\beta_{i} w_{j}^{k_{i}}\right] & (j=\mu+1, \mu+2, \cdots, n),
\end{array}\right. \\
& \Delta(\lambda)=-\prod_{i=1}^{n} \rho^{k_{i}} \cdot \prod_{j=\mu+1}^{n} e^{\rho w_{j}(b-a)} \cdot\left(\left[\theta_{0}\right]+e^{\rho w_{\mu}(b-a)}\left[\theta_{1}\right]\right) .
\end{aligned}
$$

We introduce these expressions into (10) where the elements of the last column of $N$ have been modified to the form (54). The factors of the denominator $\Delta(\lambda)$ we can divide into the numerator as follows: the factor -1 into the last column, the factor $\rho^{k_{i}}$ into the $(i+1)$-th row, the factor $e^{\rho w_{j}(b-a)}$ into the $j$-th column, the remaining factor $\bar{\Delta}$ into the $\mu$-th column. We thus obtain 


$$
G(x ; s ; \lambda)=\frac{1}{n \rho^{n-1}} \times
$$

$$
\begin{aligned}
& e^{\rho w_{1}(x-a)}[1] \cdots \frac{e^{\rho w_{\mu}(x-a)}[1]}{\left[\theta_{0}\right]+e^{\rho w_{\mu}(b-a)}\left[\theta_{1}\right]} \cdots e^{\rho w_{n}(x-b)}[1] \quad \sum_{i=1}^{\mu} e^{\rho w_{\delta}(x-\delta)}\left[w_{i}\right] \\
& {\left[\alpha_{1} w_{1}^{k_{1}}\right] \cdots \frac{w_{\mu}^{k_{1}}\left(\left[\alpha_{1}\right]+e^{\rho w_{\mu}(b-a)}\left[\beta_{1}\right]\right)}{\left[\theta_{0}\right]+e^{\rho w_{\mu}(b-a)}\left[\theta_{1}\right]} \cdots\left[\beta_{1} w_{n}^{k_{1}}\right] \quad \sum_{j=1}^{\mu} e^{\rho w_{j}(b-a)}\left[\beta_{1} w_{j}^{k_{1}+1}\right]} \\
& -\sum_{j=\mu+1}^{n} e^{\rho w_{j}(n-8)}\left[\alpha_{1} w_{j}^{k_{1}+1}\right] \\
& {\left[\alpha_{n} w_{1}^{k_{n}}\right] \cdots \frac{w_{\mu}^{k_{n}}\left(\left[\alpha_{n}\right]+e^{\rho w_{\mu}(b-a)}\left[\beta_{n}\right]\right)}{\left[\theta_{0}\right]+e^{\rho w_{\mu}(b-a)}\left[\theta_{1}\right]} \cdots\left[\beta_{n} w_{n}^{k_{n}}\right] \quad \sum_{j=1}^{\mu} e^{\rho w_{j}(b-s)}\left[\beta_{n} w_{j}^{k_{n}+1}\right]} \\
& -\sum_{j=\mu+1}^{n} e^{\rho w_{j}(n-s)}\left[\alpha_{n} . v_{j}^{k_{j}+1}\right]
\end{aligned}
$$

From this form of $G$ we can easily determine the limit of $I_{A B}^{n-x}$.

Assume the sequence of circles so chosen that their distance from the nearest point $\rho_{\mathrm{Il}}, \rho_{\mathrm{II} l}$ is at least $d>0 . *$ Then on $A . B$ when $|\rho|$ is large

In fact if

$$
\left[\theta_{0}\right]+e^{\rho w_{\mu}(b-a)}\left[\theta_{1}\right]>M>0 .
$$

$$
\left[\theta_{0}\right]+e^{p v_{\mu}(b-a)}\left[\theta_{1}\right]=\phi,
$$

where $|\phi|$ is small, we infer at once that the point $\rho$ lies near to $\rho_{\mathrm{Il}}$ or $\rho_{\mathrm{IIl}}$.

In view of (57), every element of $G$ given by (56) is bounded on $A B$ since the exponents have negative or zero real parts [see (29) and (49)]. Further, when this expression for $G$ is used in $I_{A B}^{a-x}$ the factor $n \rho^{n-1}$ cancels.

Consider those terms of $I_{A B}^{a-x}$ which do not contain the first element of the last column; $s$ is confined to this column. When we integrate these terms as to $s$, they have the form $M / \rho$ ( $M$ bounded), as an integration by parts between the points of discontinuity of $f(s)$ shows. But these terms all contain as factor an element of the first row, not the last element, beside other bounded elements ; since these first row terms are small for $|\rho|$ large, except the $\mu$-th one when $\rho$ is near to $B, \dagger$ we conclude that the $\rho$-integration of them will yield only small terms. $\ddagger$

* This refers to the minimum distance of $\rho_{\mathrm{II} l}, \rho_{\mathrm{II} l}$ from any point of the circle. In the proof of the theorem of $\xi_{1}, \rho_{\mathrm{I} l}, \rho_{\mathrm{II} l}$ (corresponding to $\lambda_{\mathrm{I} l}, \lambda_{\mathrm{II} l}$ ) were found to be approximately equally spaced along the bisectors of $S_{1}$ and $S_{2}$. The above construction of a sequence of circles is there. fore possible.

$\dagger$ Then $\rho w_{\mu}$ is a pure imaginary.

$\ddagger$

$$
\left|\int_{A}^{B} \frac{\varepsilon}{\rho} d \rho\right|<\operatorname{maximum} \text { of } \varepsilon \times \frac{\pi}{2 n} .
$$

This is small everywhere on $A B$ if $\varepsilon$ is small. Even if $\varepsilon$ is small except for a little part of $A B$, this integral is small. 'Small' means of course indefinitely small as $|\rho|$ becomes indefinitely large. 
Accordingly we need only consider the terms which contain the first element of the last column. This element has a coefficient 1 , as it had originally in $G(x, s ; \lambda)$. Here again we can restrict ourselves to terms in the brackets which do not contain $1 / \rho$. Finally therefore

$$
I_{A B}^{a-x}=\frac{1}{2 \pi \sqrt{-1}} \int_{A}^{B} \int_{a}^{x}\left(\sum_{i=1}^{\mu} e^{\rho w_{f}(x-s)} w_{i}\right) f(s) d s d \rho+\epsilon,
$$

where $\epsilon$ signifies a quantity which tends to 0 as $|\rho|$ increases.

Integrating by parts we find

$$
\int_{a}^{x} e^{\rho w_{i}(x-s)} w_{i} f(s) d s=\frac{f(x-0)}{\rho}+\frac{\epsilon}{\rho} \quad(i=1,2, \cdots, \mu),
$$

where $\epsilon$ is certainly small unless $i=\mu$ and $\rho$ is near to $B$. From this we conclude that

$$
\int_{A}^{B} \int_{a}^{x} e^{\rho w_{i}(x-s)} w_{i} f(s) d s d \rho=\frac{\pi \sqrt{-1}}{2 n} f(x-0)+\epsilon,
$$

and therefore

$$
I_{A B}^{a-x}=\frac{\mu}{4 n} f(x-0)+\epsilon .
$$

To treat $I_{B C}^{a-x}$ we multiply the columns 1 to $\mu-1$ of $N(x, s ; \lambda)$ by $+\frac{1}{2} \bar{y}_{i}(s)$, and the columns $\mu$ to $n$ by $-\frac{1}{2} \bar{y}_{i}(s)$ and add them to the last column. The elements of the $\mu$-th column can be written in this case

$$
\frac{e^{\rho w w_{\mu}(x-b)}}{e^{\rho w_{\mu}(a-b)}\left[\theta_{0}\right]+\left[\theta_{1}\right]}, \quad \frac{w_{\mu}^{k_{i}}\left(e^{\rho w_{\mu}(a-b)}\left[\alpha_{i}\right]+\left[\beta_{i}\right]\right)}{e^{\rho w_{\mu}(a-b)}\left[\theta_{0}\right]+\left[\theta_{1}\right]} \quad(i=1,2, \cdots, n),
$$

and all the elements are bounded as before. The important term again comes from the first element of the last column, and we find precisely as before that

$$
I_{B C}^{a-x}=\frac{\mu-1}{4 n} f(x-0)+\epsilon .
$$

Adding (58) and (59) we find

and by symmetry

$$
I_{\gamma_{1}}^{a-x}=1 f(x-0)+\epsilon,
$$

$I_{\gamma_{3}}^{a-x}=1 f(x-0)+\epsilon, \quad I_{\gamma_{1}}^{x-b}=1 f(x+0)+\epsilon, \quad I_{\gamma_{3}}^{x-b}=1 f(x+0)+\epsilon$.

Hence we find

$$
I_{\Gamma}^{a-\iota}=\frac{f(x-0)+f(x+0)}{2}+\epsilon .
$$

This proves the first part of the theorem.

When $x=b$ the preceding work must be modified, since now the elements 
$\mu+1$ to $n$ of the first row in (56) have the form [1]. Other elements of the last column now become important beside the first. An integration by parts with respect to $s$ followed by a $\rho$-integration gives us terms

$$
b_{1} f(a+0)+b_{2} f(b-0) \text {. }
$$

The same result must of course hold at $x=a$.

When $n=2 \mu$ the attack is almost the same. One obtains a determinant expression for $G$ by modifying the last column and distributing the two factors of $\bar{\Delta}$ into the $\mu$-th and $(\mu+1)$-th column.* The elements are bounded on $\gamma_{1}$ in this case and one obtains first $I_{\gamma_{1}}^{a-x}$ which tends toward $\frac{1}{2} f(x-0)$. The remainder of the proof is as before.

* See (38) in which $\bar{\Delta}$ is factorable into

$$
\left(\left[\phi_{1}\right]+e^{\rho w w_{\mu}(b-a)}\left[\psi_{1}\right]\right)\left(\left[\phi_{2}\right]+e^{-\rho w w_{\mu}(b-a)}\left[\psi_{2}\right]\right) .
$$

